\title{
Remotely Assessing Symptoms of Parkinson's Disease Using Videoconferencing: A Feasibility Study
}

\author{
Tereza Stillerova, ${ }^{1}$ Jacki Liddle, ${ }^{2}$ Louise Gustafsson, ${ }^{1}$ Robyn Lamont, ${ }^{2}$ and Peter Silburn ${ }^{2}$ \\ ${ }^{1}$ School of Health and Rehabilitation Sciences, The University of Queensland, Brisbane, QLD, Australia \\ ${ }^{2}$ Asia-Pacific Centre for Neuromodulation, Queensland Brain Institute and UQ Centre for Clinical Research, \\ The University of Queensland, Brisbane, QLD, Australia
}

Correspondence should be addressed to Tereza Stillerova; teza133@hotmail.com

Received 5 August 2016; Revised 29 November 2016; Accepted 7 December 2016

Academic Editor: Jeff Bronstein

Copyright (C) 2016 Tereza Stillerova et al. This is an open access article distributed under the Creative Commons Attribution License, which permits unrestricted use, distribution, and reproduction in any medium, provided the original work is properly cited.

\begin{abstract}
Purpose. To evaluate the feasibility of assessing a person's symptoms of Parkinson's disease (PD) in their home using the videoconferencing technology they already possess, without a home visit. Method. Eleven participants with PD completed the Movement Disorder Society Unified Parkinson's Disease Rating Scale (MDS-UPDRS) face-to-face and then via videoconferencing within a two-week period. Participants used free software and the computers and webcams available at their home to complete the videoconference assessment with a clinical rater scoring remotely. Clinical raters and participants provided feedback on the experience. Results. Excluding rigidity and postural stability, between zero and seven items could not be completed in the assessment of each participant (median 2.0, IQR 1.0-4.0). Between face-to-face and videoconference assessments, the median difference in scores was 3.0 (IQR 1.5-9.0). Content analysis of feedback identified the clinical raters' reasons why some scoring could not be completed and the participants' hope for future clinical application. Conclusions. In using free everyday technology available in participants' homes, MDS-UPDRS ratings could be obtained without an initial home visit; however some items were unable to be scored for some participants. Use of a protocol or technological advances are likely to reduce missing items.
\end{abstract}

\section{Introduction}

Parkinson's disease (PD) is a progressive, neurodegenerative disorder that is characterised by disordered movement including bradykinesia (slowness of movement), resting tremor, rigidity, and postural instability [1]. Whilst motor symptoms define the disorder, extensive nonmotor symptoms such as mood disorders, sleep problems, and autonomic dysfunction are also present, making it important to assess both motor and nonmotor symptoms [2]. High-frequency monitoring through reassessment is required due to the progressive nature of PD and the occurrence of many shortterm variations in symptoms [3]. To get treatment regimens correct, monitoring is important to refine the trade-off between maximising the opportunity for the medication to cause significant changes in symptoms and minimising drug exposure to reduce the risk of side effects [3].

As the second most common neurodegenerative disorder, it has been projected that by 2030 the prevalence of PD will have, at a minimum, doubled since 2005 in the most populous nations [4]. This increase in prevalence is expected to increase the burden on health resources, which may adversely affect a client's access to care and monitoring [4]. Another factor affecting access to monitoring is a client's geographical location and the distribution of neurologists in remote areas [5]. In rural Australia, waiting for a neurologist's appointment may delay dosage manipulation by 6 to 12 months [6]. This means clients with PD may be unnecessarily living with side effects, such as sedation or dyskinesia, for extended periods of time. With timely monitoring, many of these adverse effects are reversible through reduction or cessation of medication [7]. As PD progresses, loss of mobility can cause clients to become bedridden or confined to a wheelchair, increasing the difficulty of travelling to medical appointments [8].

Given the resource intensive nature of frequent faceto-face appointments for clients and services, the remote monitoring of PD symptoms has been explored [9-13]. 
Research has found that conducting the UPDRS through videoconferencing is reliable and valid in comparison to administering the assessment in person and is a cost-effective and efficient alternative to in-person monitoring [9-13]. However, typically these videoconference assessments were not conducted in the home environment, with clients being tested at a nominated site within their community or a dedicated testing room. Additionally, clients had access to readily available technicians, clinical raters, or nurses who assisted with or conducted the clinical examination, which was also assessed by an additional health professional at a remote location $[9,11,12]$. Despite presenting positive results, previous studies have used specialised and often expensive equipment or software, in addition to having staff present when administering Internet-based assessments. This makes results difficult to generalise to the wider PD population who may be unable to reproduce these setups or may not be in a position to wait for the delivery of equipment or availability of support staff $[9,11-13]$. Although items that need to be physically assessed would not be possible to conduct virtually, there is a need for a true home-based assessment, to provide people with PD a cost-effective and timely option for showing their health professional new symptoms, without waiting for specialised telehealth equipment to be delivered and without a health professional present.

Abdolahi et al. (2013) conducted a study to compare whether administering the UPDRS without the items that require physical interaction would make a significant difference to trial outcomes [8]. Modified UPDRS scores, which exclude the rigidity and postural stability testing items, were compared to the standard UPDRS [8]. The results indicated the same clinical outcomes were demonstrated with the modified scores, and a high internal consistency was found. The authors suggested that the modified UPDRS could lay foundations for remote assessment as all of the items could be completed visually through videoconferencing. However, the modified UPDRS was only elicited statistically from scores from in-person clinic-visit assessments.

The purpose of this study was to evaluate the feasibility of remotely assessing the symptoms of PD in a person's own home using readily available technology, specifically a webcam and a freely available Internet-based videoconferencing option, such as Skype ${ }^{\mathrm{TM}}$ or Google Hangouts without a preparatory home visit. It also investigated the participants' and clinical raters' experiences of using Internet-based assessment and any limitations that were encountered.

\section{Method}

2.1. Participants. This study was conducted with the approval of the UnitingCare Health Human Research Ethics Committee (number 1312) and Behavioural and Social Sciences Ethical Review Committee, The University of Queensland (number 2013000913). Participants were recruited through research networks and $\mathrm{PD}$ organisations. The attrition rate was zero. The participant inclusion criteria included a diagnosis of PD, the availability for two appointments one to two weeks apart, and access to suitable technology. Participants who could not be seen face-to-face for the first appointment were excluded from this study. The purpose and procedures of this study were explained to each participant in writing prior to obtaining written consent. Participants were not paid to participate in the study; however the cost of parking for clinic attendance for the first appointment was covered.

2.2. Measures. A demographic questionnaire was used to gather information about the participant's age, gender, marital status, ethnicity, care received, living situation, postcode, comorbidities, their PD diagnosis, and medication. The Movement Disorder Society Unified Parkinson's Disease Rating Scale (MDS-UPDRS) was used to measure the symptomatology experienced by participants, initially face-to-face and then via video conferencing [14]. The MDS-UPDRS was utilised as it is the most extensively used and comprehensive assessment of motor and nonmotor symptoms of PD [15]. The MDS-UPDRS measures PD symptoms divided into four categories: nonmotor experiences of daily living, motor experiences of daily living, motor examination, and motor complications. All four of the MDS-UPDRS subscales have five uniform response options of 0 (normal), 1 (slight), 2 (mild), 3 (moderate), and 4 (severe). A higher final score indicates higher severity and impact of PD symptoms on the person's function.

Feedback forms were used to collect information about participant experience and concerns regarding their experience of using Internet-based videoconferencing to assess their symptoms. Open and closed questions were used in the feedback form to develop an understanding of the participant's perception of how accurately videoconferencing reflected their symptoms, experiences, and concerns about the process and perceived advantages. Closed questions such as "do you think monitoring the symptoms in this way accurately reflects your current functioning?" were followed by open questions of "can you explain why?" to allow participants to elaborate on their responses. The questions were purpose-designed for the feedback forms and were developed from initial consumer consultation in the development of the project. Clinical rater feedback was collected by asking raters to provide written reflection on their experiences of assessing participants through videoconference, immediately after each session ended.

2.3. Equipment. Videoconferencing software programs that were readily available, user friendly, and free to download and use, including Skype or Google Hangouts, were options for the videoconferencing part of the study. As preparatory home visits were not conducted, participants used the computers and webcams that they already had at home when engaging in the videoconferencing assessment.

2.4. Procedures. This research was designed as an exploratory, feasibility study. Four clinical raters, consisting of qualified occupational therapists and physiotherapists, were involved in data collection. Clinical raters completed the MDSUPDRS training video and also observed at least one session with an experienced rater. To reduce discrepancies between assessments, the same clinical rater was allocated to conduct a participant's face-to-face and videoconference assessment 
where possible. During the first appointment, the participants filled in the demographic questionnaire and had their PD symptomology assessed in person, either in their home or within a private clinic room, using the MDS-UPDRS. At the end of the face-to-face session, the participants were given an envelope containing the MDS-UPDRS self-report questionnaire, a feedback form, and return envelope, for their videoconference assessment. An Internet-based videoconference appointment was arranged for approximately one to two weeks later at the same time since dopaminergic medication so that the effect of medications would be consistent and because participants were expected to stay relatively stable over the one- to two-week period.

In the second session, either Skype or Google+ Hangouts was used to conduct the videoconference assessment, with the participant using their home computer and webcam. The clinical rater, located remotely from the participant, scored the MDS-UPDRS, excluding the postural stability and rigidity testing. Participants were given the option of independently completing the self-report components or answering the questions verbally via videoconferencing if writing was burdensome given their symptoms. For some items on the MDS-UPDRS, such as items that required observations of foot movement or parts of the body that were not naturally in the frame of the videoconference, clinical raters requested that participants repositioned their webcams or laptops if possible and safe. Participants were then asked to complete the feedback form and mail back all their documents in the return envelope.

2.5. Data Analysis. This was a feasibility study and hypothesis testing was not conducted. Descriptive statistics were used to report the sample demographics. The postural stability and rigidity testing scores were excluded from the analysis. Graphs were generated in Microsoft 2007 Excel $^{\circledR}$ to demonstrate the frequency of missing data and score differences between face-to-face and videoconference assessment. The number of missing items for each participant was also calculated to explore the median and range for the missing data.

A content analysis was utilised to organise the open response data from participant and clinical rater feedback [16]. Through a process of identifying content categories which arose from the data, data were then classified as belonging, or not belonging, to a particular category. This process generated a means of describing the main content within the feedback to enable understanding of the participants' and clinical raters' experiences with remote assessment. The categorisation was independently checked by a second researcher.

The round trip travel time and distance savings for clinical raters and participants were calculated using Google Maps ${ }^{\odot}$. In the first face-to-face appointment, participants or clinical raters travelled between the private clinic and the participant's home. The starting location entered into Google Maps was the participant's postcode, obtained in the demographic questionnaire, and the destination was standardised as the University of Queensland Centre for Clinical Research.

\section{Results}

Eleven participants were included in this study. The participants were seven men and four women with a median age of 69.0 years (57.0-76.0), living in Queensland, Australia. The median age of diagnosis of PD was 59.0 years (IQR 54.071.5 ), and the median number of years since diagnosis was three years (IQR 2.5-9.5). The MDS-UPDRS indicated that the sample's symptom severities ranged from slight to severe, with most participants displaying slight-moderate symptoms. Nine out of the 11 participants were taking dopaminergic medication for their PD. Two participants were recipients of deep brain stimulation treatment. Seven out of 11 participants received informal care from family or friends, and two out of 11 received care from a community or residential service.

During the videoconferencing assessment, ten participants used Skype, and one participant used Google+ Hangouts. Two participants used a Smartphone or Tablet device and the remaining nine used a computer.

3.1. Frequency of Missing Data. There was no missing data for the self-report MDS-UPDRS subscales of motor and nonmotor symptoms and motor complications. In the motor examination of each participant, between zero and seven items could not be completed, excluding the intentionally omitted items of rigidity and postural stability. The median number of items missing for each participant was 2.0 (IQR 1.0-4.0). The frequency of the missing items that could not be completed is demonstrated in Figure 1. Whilst rigidity and postural stability were expected to have a frequency of 11, due to intentional omission caused by the inability to conduct physical tests for the 11 participants over videoconference, rest tremor of the lower extremity had a similarly high missing item frequency of 10 .

From the clinical rater feedback, two key content areas emerged indicating reasons for missing items. The first, most commonly reported content category, was positioning. Clinical raters reported they were unable to rate some items for some participants due to the inability to see the participants' entire bodies because of space constraints. Clinical rater 1 reported that "the participant's office was too small for them to move away from the camera and demonstrate gait, or allow me to observe their lower limbs." In some cases, clinical raters reported being able to brainstorm with participants to achieve a better vantage point for observation, including using laptops in large open areas so that participants could move away from their webcam enabling their whole body to be seen. It should be noted however that some participants had desktop computers in small rooms that raters felt would be unsafe for participants to move independently to another location. The videoconferencing image frame also impacted on the importance of participant positioning. Clinical raters had "limited vision of whole body perspective" during the interview, causing difficulties in rating items that are intended to be observed throughout the interview such as global spontaneity of movement and constancy of rest tremor. Clinical raters made every effort to safely score all possible items, using the space and equipment available at the participant's home. Clinical raters noted that although some participants 


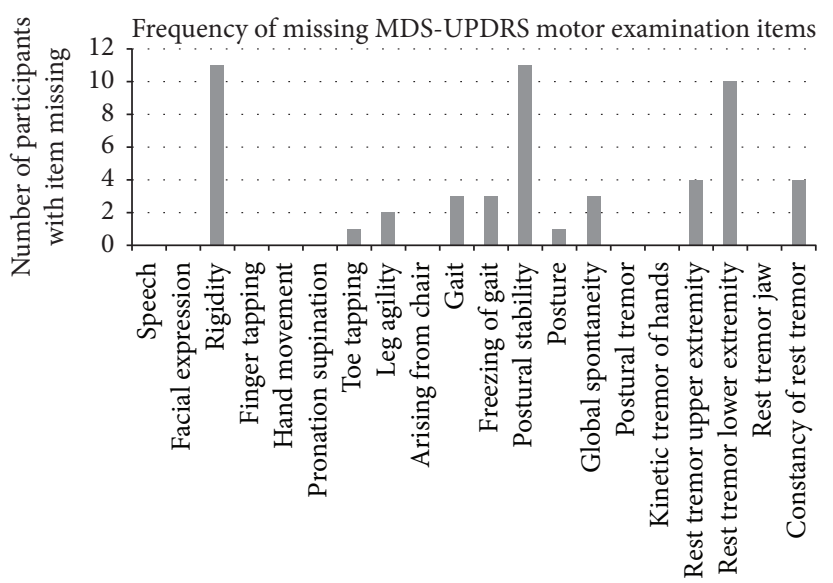

Motor examination items

FIGURE 1: Frequency of missing MDS-UPDRS motor examination items during the Internet-based videoconference assessment of 11 participants.

indicated willingness to bring lower limbs into the frame (e.g., by placing feet on a chair), there were concerns that this would be unsafe and an invalid indication of general movement patterns. Webcams inbuilt into computers were unable to be easily manipulated so lower limbs could not be assessed for participants with this setup.

The second major content category explaining missing items was technical difficulties. Internet connections made the images pixelated for two assessments, causing difficulties scoring motor examination items where the criteria for scoring depends on the rhythm and fluency of the participants movement, including finger tapping, hand movements, pronation-supination of hands, leg agility, and toe tapping. Clinical rater 2 reported that they needed "to ask (the participant) to slow down as vision of action was blurry" during the finger tapping task. Postural tremor of the hands, kinetic tremor, and rest tremor amplitude items require an indicator of the magnitude of tremor in centimetres. Clinical rater 3 reported that clinical raters would "need calibration to allow rating of kinetic tremor...for example a ruler as a target" for accuracy.

3.2. Difference in Scores. The difference in scores between the face-to-face and the videoconference assessment for the four subscales of the MDS-UPDRS are represented in Figure 2 in the direction in which they occurred. Negative score difference represents that PD symptoms were rated as more severe during videoconference compared to faceto-face assessment. The biggest differences occurred in the motor examination, with the median difference between scores being 3.0 (IQR 1.5-9.0) out of possible 132 points for this subscale. Within the motor examination, the biggest differences between face-to-face and videoconference scores were for participants 8,9 , and 11 who, respectively, had a difference of 18,26 , and 14 points out of possible 132 points. This did not reflect their differences in scores in the other three self-report subscales completed at the same time.
3.3. Participant Feedback Content Analysis. Participant feedback indicated that the majority of participants, 10 out of 11, rated their experience with using videoconferencing to monitor their PD symptoms as good, and one out of 11 participants rated it as excellent. No participants indicated a neutral or negative experience. The majority of participants, eight, identified with being "quite familiar" or "very familiar" using their respective videoconferencing software, and one participant identified with being "neither familiar nor unfamiliar" and two identified with being "very unfamiliar."

Participants identified both concerns and advantages. Within concerns, two key content categories emerged: current limits to technology and concern for others' experiences. Within the first content category, participants identified awareness of clinical raters' inability to monitor all aspects of their motor performance without changing computer positioning within the home environment. As participant 9 stated, "It's difficult to see everything that is happening due to space." Another issue regarding technology was raised by one participant, questioning whether interactions monitored over videoconferencing would stay "secure and private" (participant 10). In the second content category, despite reporting positive experiences for themselves, participants expressed concerns that others may not have such easy access. In particular, there were concerns for patients unfamiliar with, or without access to, computers.

The most frequently reported feedback was the convenience and resource efficiency that videoconferencing permitted. Participant 9 reported, it "saves the travel to see the doctor," whilst participant 1 stated that reduced travel makes monitoring "less stressful." Participant 11 also reported that it would be an advantage to see a neurologist more efficiently if "displaying symptoms that were intermittent" which may not be present during their clinic visit. The perceived advantages of using videoconferencing to monitor symptoms were more commonly reported than disadvantages. The overall perception of participants was that this was a good way of monitoring symptoms. Participants identified feeling positive 


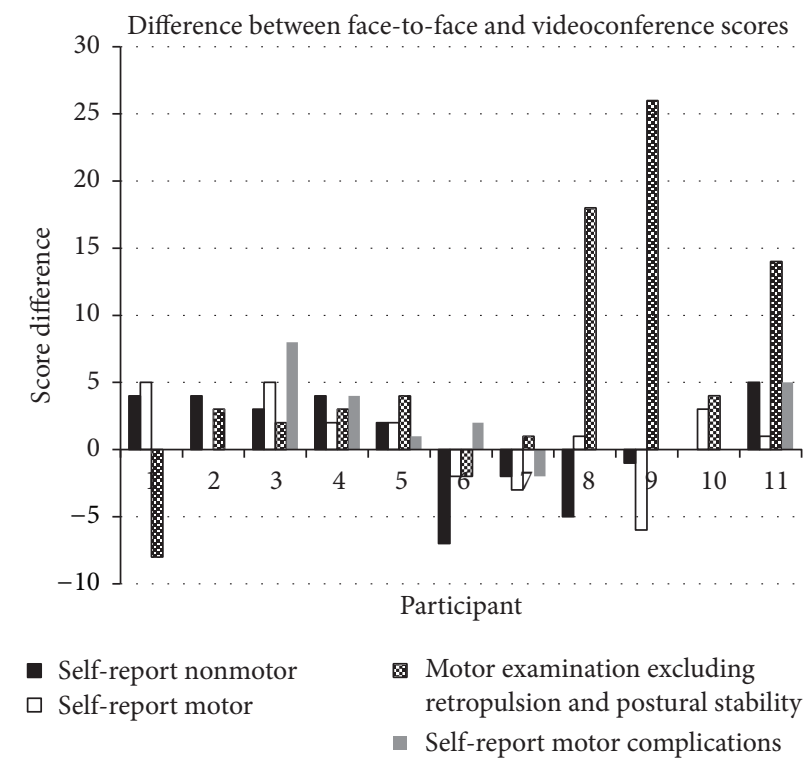

FIGURE 2: Difference between face-to-face and Internet-based videoconference assessment scores per participant for each MDS-UPDRS subscale.

about interacting with the clinical rater via teleconference, as reported by participant 10 "it felt comfortable communicating in this manner."

3.4. Resource Savings. In the first face-to-face appointment, participants or clinical raters travelled between the clinic and the participants home, depending on whether the clinical rater was coming to assess the participant in their own home or if the participant was coming to the clinic. The median travel time and travel distance savings when remotely assessing were 86.0 minutes (IQR 38.0-226.0) and 60.2 kilometers (IQR 24.3-278.0) per participant, respectively.

\section{Discussion}

Parkinson's disease is a condition with high symptom variability that requires frequent monitoring. It was known from previous research that a modified version of the UPDRS excluding rigidity and postural stability testing was reliable and valid, laying the foundations for its use in remote assessment of research participants and clients [8].

This feasibility study was conducted in the present context of extensive neurologist waiting lists and lack of geographical dispersion of neurologists [5], to determine whether a readily available option that did not require specialised technology could be utilised by clients. Clinically, this would provide an option for showing health professionals as soon as new symptoms arose without the burden of travelling or the potential that a transient symptom may not be evident during an appointment. Overall, the results from this study suggest that the provision of guidelines for a suitable environmental setup, including distances from the camera and requirement of a walking area, could take place prior to assessments to improve the clinical raters' ability to score the assessment. In the existing literature, ways to reduce missing values have been described; the Connect. Parkinson study performed a test connection with participants prior to actual consultations to overcome such barriers in advance [17]. As this method does not require in-person support, it is consistent with the aims of this study to provide consultations without preparatory home visits. Missing items may be of particular concern to people whose prominent symptoms are rigidity or postural stability related. Additionally, resting tremor was a frequently missing item in this study, so careful consideration of the person's symptomology, and whether videoconference would capture it, would be needed before using this method. Using equipment that people already have at home may enable more cost-effective and timely monitoring than options which require mailing equipment or home visitation by clinical staff. The distance and time savings when videoconferencing could be considered when designing and delivering services, as high satisfaction with the videoconferencing assessment in this study appeared to relate to participants not having to travel to appointments and associated time and cost savings. This is consistent with findings from Wilkinson et al. (2016) who reported greater satisfaction in patients with PD using telehealth in terms of convenience and accessibility/distance and reduced travel burden using telehealth [18]. Previous literature also supports higher client satisfaction with Internet-based UPDRS assessment compared to faceto-face assessment [10]. The results from this study provide supportive evidence for high satisfaction rates with their experience of Internet-based videoconference assessments, although participants were not asked whether they prefer these to face-to-face assessments.

The results reveal how the scores differ between the faceto-face and videoconference assessment, and they suggest that symptoms additional to rigidity and postural stability are difficult to monitor in this medium. The self-report questions in the UPDRS ask participants to consider their symptoms 
over the past week, whilst the motor examination is based on how their symptoms are at that point in time. This means the scores are expected to vary over time given the oftenturbulent nature of PD. Additionally, the motor examination is the only part of the MDS-UPDRS that relies on examiner observation, not self-report; thus it is expected that scores for this subscale will vary the most. Overall, the difference between face-to-face and videoconference scores for the motor examination appear to be the same magnitude as the score differences in the other three MDS-UPDRS sections, which may reflect the variability in PD symptoms, as opposed to variability caused by the videoconference medium.

Three participants demonstrated motor examination score differences inconsistent with the score differences in the other three self-report subscales. Participants 8 and 9 self-report motor complication scores were the same between assessments and their self-report nonmotor was reported to be worse during the videoconference assessment. Participant 11's self-report scores are in the same direction as their motor examination, however not the same magnitude. The reason behind this difference is not clear as the time since medication was taken, medication type taken, and clinical rater stayed constant between both assessments. However, the impact of particular symptoms and the array of symptoms differed substantially between people with $\mathrm{PD}$, and there are many factors that impact on PD symptoms outside of time since medication, such as comorbidities, general health, and psychosocial factors [19].

Previous studies investigating the feasibility of online assessment have identified that PD symptoms such as hypophonia (soft voice) may cause clinical raters difficulty in hearing the participant clearly, and symptoms of tremor, rigidity, dyskinesia, and bradykinesia may cause the participants difficulty using computers when needing steady hand movements to navigate a mouse $[12,20]$. The results from this study, from the content analysis of clinical rater and participant feedback, did not indicate that PD symptoms impacted on the participant's ability to engage in any aspects of the videoconference assessment. Rather the predominant reason for missing items in this study was the inability to capture the participant's whole body in the videoconference frame, therefore making it difficult to score some items of the MDS-UPDRS. Items that could be completed were items that could occur naturally within the frame of the videoconference throughout the assessment, without the participant having to change the webcam's positioning. The items that could not always be completed all required the webcam's position to be changed, or for the participant's whole body to be seen throughout the assessment, which does not occur naturally in an Internet-based videoconference frame. This could be overcome by asking participants to be located in rooms large enough for them to walk across and to enable clinical raters to observe their lower limbs. The quality of technology used in previous studies, including webcam quality and strength of Internet connection, has also been shown to impact the reliability and validity of online monitoring $[10,13]$. Consistent with this, the results from this study revealed that clinical raters experienced technological difficulties in the motor examination items within the MDSUPDRS due to videoconference image pixelation, freezing, and disjointedness, especially if the movement performed was fast.

A protocol and guidelines should be developed for future studies to reduce the amount of missing items. No specific direction was given about computer setup in this study, but standardised setups from other telehealth processes and established telehealth guidelines could be applied to this situation. For example, the American Telemedicine Association's Home Telehealth Clinical Guidelines promote providing service users with clear, simplified written information, diagrams and pictures to facilitate the appropriate placement and use of technology [21]. Based on the findings from the present study, recommendations for a protocol include webcam positioning guidelines and suggesting participant location in open spaces such as living rooms so they can move back from the camera and have their whole body seen for the entirety of the assessment. As technology options expand, the protocol should also consider positioning for a variety of different devices such as phones, tablets, laptops, and computers. Privacy should be protected by ensuring health professionals are not in an open-plan office and are wearing headphones during the Internet-based videoconferencing assessment. In addition, the use of specialised medical teleconference software that operates similarly to Skype and could be downloaded onto existing hardware at no patient cost should be considered. Whilst Skype is regarded as acceptable within the Australian context by government and professional bodies $[22,23]$ specialised software (for example eHAB) can ensure heightened security, reducing concerns of participants, and also allow for store and forward capacity to improve quality of video received. Outside Australia, teleconference users must check the security standards required to have communication with patients. Whilst practical safety considerations should also be integrated, the items on the MDS-UPDRS are not beyond what people would normally do in their home environment.

4.1. Study Limitations. A limitation of this study was that a clinical rater scored both the face-to-face and videoconference assessments of a participant, which could have resulted in the clinical rater carrying knowledge from the face-to-face assessment into the videoconference assessment. Although this is not desirable in a research setting, clinically this could be an advantage because if a clinical rater is familiar with their client's symptoms, which would often be the case within clinical teams, they may be able to observe changes in symptoms more easily. Participant self-selection was another limitation, as participants who felt more comfortable using technology may have been more likely to volunteer their participation for this study. Sample size was also reflective of the purpose of this study being a feasibility study. However, some clear issues consistently surfaced after 11 participants, such as there being more missing items than the literature suggested and the videoconferencing process needing more structuring. It was therefore determined that the results from the initial 11 participants and four examiners be analysed and recommendations be made for larger future studies. A 
limitation that may have contributed to higher missing items then expected was that clinical raters did not receive training to optimise their physical examination skills in a telehealth setting. It should also be acknowledged that clinical raters were not movement disorder specialists, which could have contributed to discrepancies of some of the data.

4.2. Future Directions. As the average age of onset of PD is the early-to-mid 60s [24], the ability of the older population to use technology must be considered. Although the majority of the participants reported that they were "quite familiar" or "very familiar" with videoconferencing, from the content analysis of participant feedback, it can be seen that participants had concerns for others who may not have access to personal computers or have adequate computer literacy. In Australia the proportion of the population older than 60 who use the Internet has almost doubled between 2003 (21\%) to 2009 (41\%), demonstrating an increase in familiarity and acceptance of using the Internet in this population [25]. This aligns with the principles of this study, which were to cater to what technology people already have in their home, instead of introducing new technology.

Future studies should consider how many people have access to this technology and show interest in this method of monitoring, and how many health professionals would be open to using this option. In the United States of America, the Connect. Parkinson study found that latent demand for virtual house calls is high, and it would be important to establish whether the demand is similarly as high in Australia [17]. Training support should be offered for optimal usage. Research found that health professionals were more satisfied with face-to-face visits and concluded that this was most likely due to technological incidents whilst scoring [10]. Whether people perform better or worse in the assessment when they are at home may also need to be investigated, as one participant in this study reported they felt doing the assessment from home was "less stressful."

\section{Conclusion}

The results of this study show that despite there being more missing items than expected, this process of monitoring PD symptoms using Internet-based videoconferencing may be useful to give an overall idea of symptom severity over time. It may also be useful in monitoring participant symptoms and reducing travel time, distance, and associated costs to see a health professional. Future research and application should develop and use a protocol which would enable fewer missing items, such as structured environmental setup. Monitoring through videoconference should be pursued further due to consumer interest and because it may provide a more immediate and better overview of a client's symptoms in between clinic visits.

\section{Competing Interests}

The authors declare that there is no conflict of interests regarding the publication of this paper.

\section{References}

[1] E. C. Hirsch, P. Jenner, and S. Przedborski, "Pathogenesis of Parkinson's disease," Movement Disorders, vol. 28, no. 1, pp. 2430, 2013.

[2] V. W. Sung and A. P. Nicholas, "Nonmotor symptoms in parkinson's disease. Expanding the view of parkinson's disease beyond a pure motor, pure dopaminergic problem," Neurologic Clinics, vol. 31, no. 3 S, p. -S16, 2013.

[3] M. Little, P. Wicks, T. Vaughan, and A. Pentland, "Quantifying short-term dynamics of parkinson's disease using self-reported symptom data from an internet social network," Journal of Medical Internet Research, vol. 15, no. 1, article no. e20, 2013.

[4] E. R. Dorsey, R. Constantinescu, J. P. Thompson et al., "Projected number of people with Parkinson disease in the most populous nations, 2005 through 2030," Neurology, vol. 68, no. 5, pp. 384-386, 2007.

[5] Federal Policy Initiatives: A New Approach to Parkinsons Disease, Parkinson's Australia Inc, Canberra, Australia, 2010, http://www .parkinsonsnsw.org.au/assets/attachments/media/PA_NewPolicyInitiative.pdf.

[6] G. F. Duncan and P. Rositano, "Parkinson's disease in regional Australia," Rural and Remote Health, vol. 11, no. 4, article no. 1658, 2011.

[7] G. Liang, "Medication adjustment after deep brain stimulation surgery of the subthalamic nucleus," in Deep Brain Stimulation for Parkinson's Disease, G. H. Baltuch and M. B. Stern, Eds., pp. 129-137, Informa Healthcare, New York, NY, USA, 2007.

[8] A. Abdolahi, N. Scoglio, A. Killoran, E. R. Dorsey, and K. M. Biglan, "Potential reliability and validity of a modified version of the Unified Parkinson's Disease Rating Scale that could be administered remotely," Parkinsonism \& Related Disorders, vol. 19, no. 2, pp. 218-221, 2013.

[9] M. Achey, J. L. Aldred, N. Aljehani et al., "The past, present, and future of telemedicine for Parkinson's disease," Movement Disorders, vol. 29, no. 7, pp. 871-883, 2014.

[10] E. Cubo, J. M. Trejo Gabriel-Galán, J. Seco Martínez et al., "Comparison of office-based versus home web-based clinical assessments for Parkinson's disease," Movement Disorders, vol. 27, no. 2, pp. 308-311, 2012.

[11] E. R. Dorsey, L. M. Deuel, T. S. Voss et al., "Increasing access to specialty care: a pilot, randomized controlled trial of telemedicine for Parkinson's disease," Movement Disorders, vol. 25, no. 11, pp. 1652-1659, 2010.

[12] T. Hoffmann, T. Russell, L. Thompson, A. Vincent, and M. Nelson, "Using the internet to assess activities of daily living and hand function in people with Parkinson's disease," NeuroRehabilitation, vol. 23, no. 3, pp. 253-261, 2008.

[13] A. Samii, P. Ryan-Dykes, R. A. Tsukuda, C. Zink, R. Franks, and W. P. Nichol, "Telemedicine for delivery of health care in Parkinson's disease," Journal of Telemedicine and Telecare, vol. 12, no. 1, pp. 16-18, 2006.

[14] C. G. Goetz, S. Fahn, P. Martinez-Martin et al., "Movement disorder society-sponsored revision of the unified Parkinson's disease rating scale (MDS-UPDRS): process, format, and clinimetric testing plan," Movement Disorders, vol. 22, no. 1, pp. 4147, 2007.

[15] Movement Disorder Society Task Force on Rating Scales for Parkinson's Disease, “The Unified Parkinson's Disease Rating Scale (UPDRS): status and recommendations," Movement Disorders, vol. 18, no. 7, pp. 738-750, 2003. 
[16] S. Elo and H. Kyngäs, "The qualitative content analysis process," Journal of Advanced Nursing, vol. 62, no. 1, pp. 107-115, 2008.

[17] E. R. Dorsey, M. A. Achey, C. A. Beck et al., "National randomized controlled trial of virtual house calls for people with Parkinson's disease: interest and barriers," Telemedicine Journal and e-Health, vol. 22, no. 7, pp. 590-598, 2016.

[18] J. R. Wilkinson, M. Spindler, S. M. Wood et al., "High patient satisfaction with telehealth in Parkinson disease," Neurology: Clinical Practice, vol. 6, no. 3, pp. 241-251, 2016.

[19] A. D. Mosley, D. S. Romaine, and A. Samii, The Encyclopedia of Parkinson's Disease, Facts on File, New York, NY, USA, 2nd edition, 2009.

[20] L. M. Cunningham, C. D. Nugent, G. Moore, D. D. Finlay, and D. Craig, "Computer-based assessment of movement difficulties in Parkinson's disease," Computer Methods in Biomechanics and Biomedical Engineering, vol. 15, no. 10, pp. 1081-1092, 2012.

[21] American Telemedicine Association, Home Telehealth Clinical Guidelines, American Telemedicine Association, Washington, DC, USA, 2003, https://hub.americantelemed.org/viewdocum/ home-telehealth-clin.

[22] MBS online-questions and answers, Canberra, ACT, Department of Health, 2012, http://www.mbsonline.gov.au/internet/ mbsonline/publishing.nsf/Content/connectinghealthservicespatients-QA\#q1.

[23] P. Hayes, "From a distance," Good Practice, no. 3, pp. 6-9, 2014.

[24] J. C. Latourelle, N. Pankratz, A. Dumitriu et al., "Genomewide association study for onset age in Parkinson disease," BMC Medical Genetics, vol. 10, no. 1, article 98, 2009.

[25] Australia Bureau of Statistics Age Matters, The Australian Bureau of Statistics, Canberra, Australia, 2011, http://www.abs .gov.au/ausstats/abs@.nsf/7d12b0f6763c78caca257061001cc588/ ea6bbe44feac7fe2ca2572a400109d19! OpenDocument. 


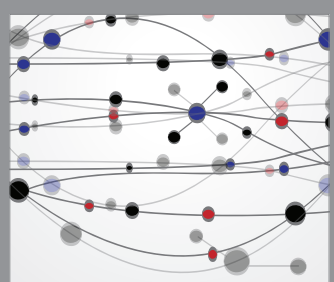

The Scientific World Journal
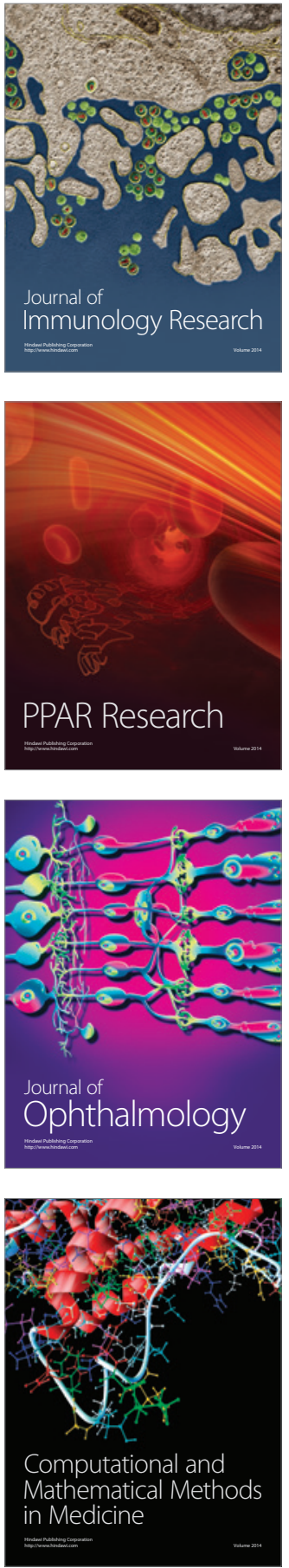

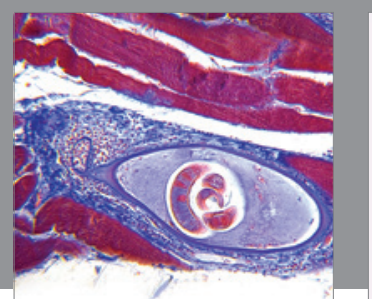

Gastroenterology Research and Practice

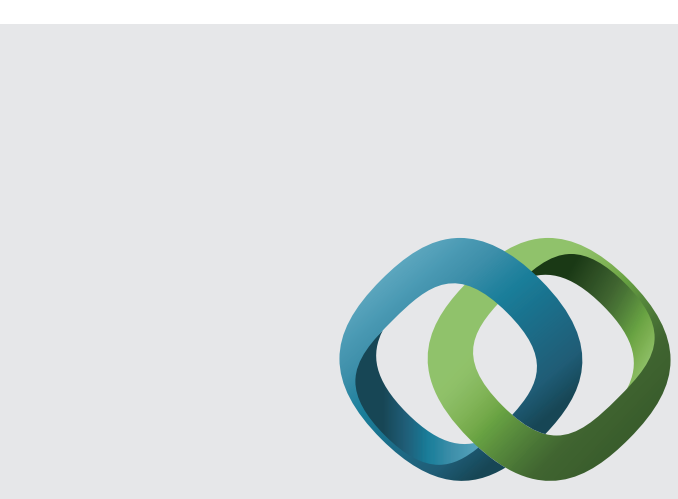

\section{Hindawi}

Submit your manuscripts at

http://www.hindawi.com
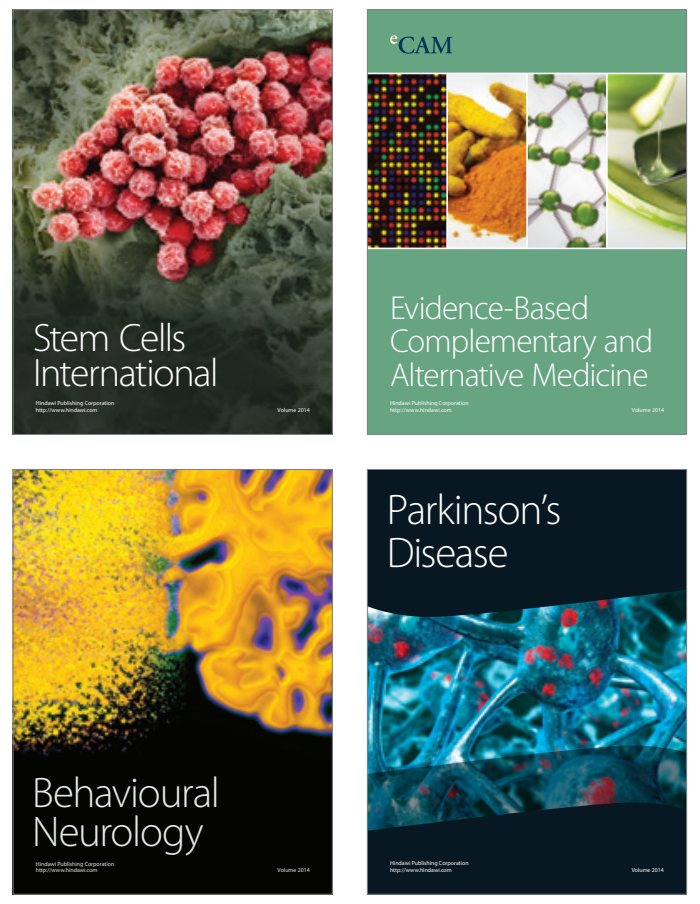
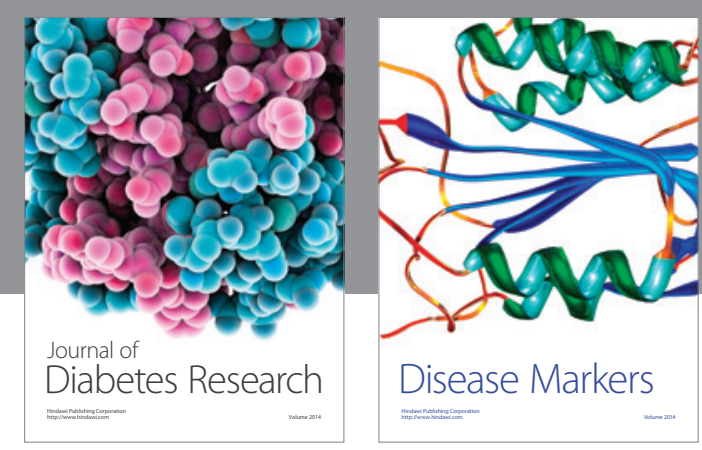

Disease Markers
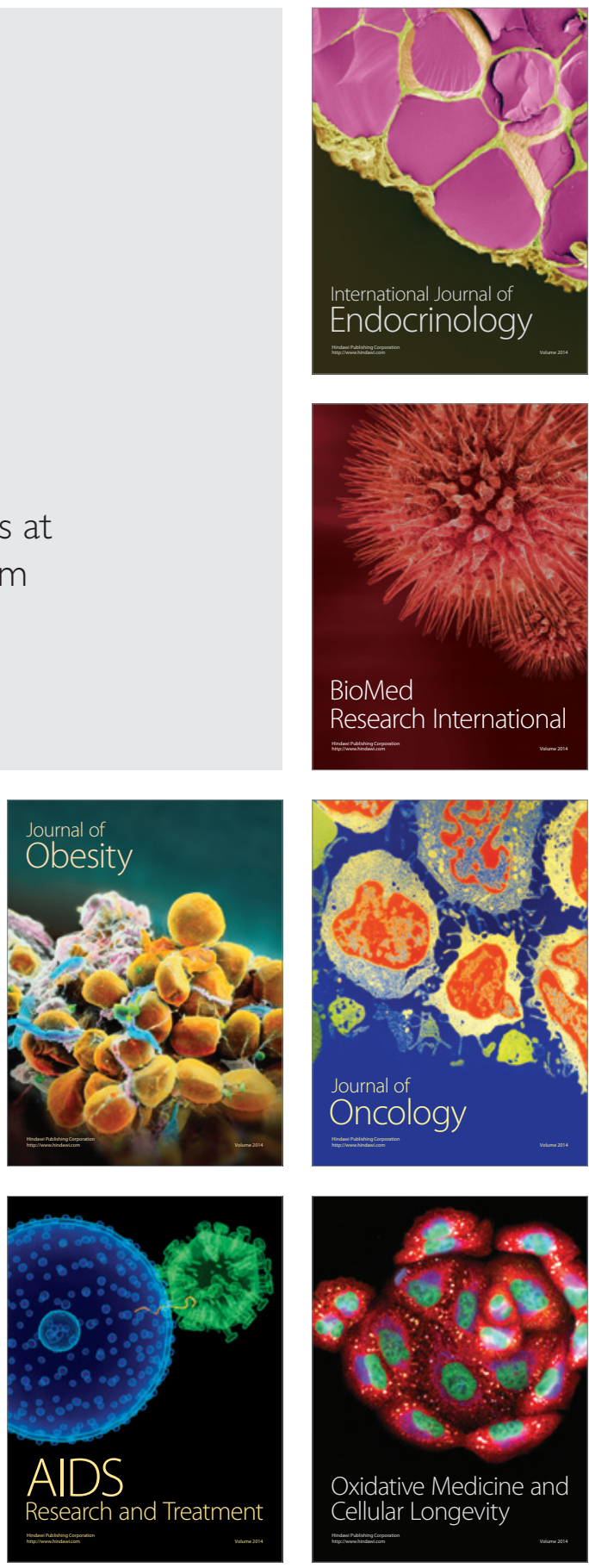